\title{
Bio-Inspired Microfluidics for Wearable Sensors ${ }^{\dagger}$
}

\author{
Chuan Nie ${ }^{1}$, Arjan Frijns ${ }^{1}$, Rajesh Mandamparambil ${ }^{2}$, Marcel Zevenbergen ${ }^{2}$ and \\ Jaap Den Toonder ${ }^{1, *}$ \\ 1 Department of Mechanical Engineering, Eindhoven University of Technology, 5600 MB Eindhoven, \\ The Netherlands; chuan.nie@merckgroup.com (C.N.); A.J.H.Frijns@tue.nl (A.F.) \\ 2 Holst Centre / Imec, 5605 KN Eindhoven, The Netherlands; rajesh.mandamparambil@nxp.com (R.M.); \\ Marcel.Zevenbergen@imec-nl.nl (M.Z.) \\ * Correspondence: j.m.j.d.toonder@tue.nl \\ + Presented at the 5th International Symposium on Sensor Science (I3S 2017), Barcelona, Spain, \\ 27-29 September 2017.
}

Published: 20 November 2017

Wearable sensors are positioned close to, on, or even inside the human body and measure vital functions such as heart rate, temperature, or even biochemical parameters. These parameters give essential information on the health and well-being of humans, and therefore wearable sensors will find applications in health monitoring, well-being, and sports.

Sweat is an interesting and convenient body fluid for wearable sensor applications. The amount of sweat and its composition can be used to detect, for example, dehydration or cystic fibrosis. To enable the continuous and non-invasive monitoring of this body fluid, we have developed a wearable sweat sensor using principles that were inspired by biology. Water transportation in plants is successfully mimicked in a flexible microfluidic system: we realized a system in which (1) liquid can be collected from the skin by an absorbing structure; (2) liquid is transported through a microchannel structure by capillarity; and (3) evaporation through a porous structure at the device outlet drives a continuous and prolonged flow through the channel (by evaporative pumping) [1]. We integrated a $\mathrm{pH}$ sensor chip in the device.

Our proof-of-concept experiments show that our prototype can be successfully used for continuous sensing [2]. It offers a base platform to integrate heterogonous sensing systems in a flexible and possibly low-cost way not only for sweat sensing but also for other applications such as continuous water quality monitoring or other bio-sensing applications where continuous flow over a sensor is required.

\section{References}

1. Nie, C.; Frijns, A.J.H.; Mandamparambil, R.; den Toonder, J.M.J. A microfluidic device based on an evaporation-driven micropump. Biomed. Microdevices 2015, 17, 47.

2. Nie, C.; Frijns, A.J.H.; Zevenbergen, M.A.G.; den Toonder, J.M.J. An integrated flex-microfluidic-Si chip device towards sweat sensing applications. Sens. Actuators B Chem. 2016, 227, 427-437.

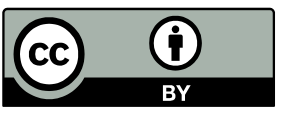

(C) 2017 by the authors. Licensee MDPI, Basel, Switzerland. This article is an open access article distributed under the terms and conditions of the Creative Commons Attribution (CC BY) license (http://creativecommons.org/licenses/by/4.0/). 